\title{
Probiotic properties of bacteria isolated from bee bread of stingless bee Heterotrigona itama
}

\begin{abstract}
Pollen collected by stingless bee is stored and packed in honey pots following the addition of various enzymes and nectar, or honey which contains microorganisms to undergo lactic acid fermentation to produce bee bread. Naturally fermented foods are known to contain probiotic bacteria. Thus, the aim of this study was to isolate probiotic bacteria from bee bread and characterize the probiotic potential of bacteria with antibacterial activities against foodborne pathogens. Bacteria were identified using 16S rRNA gene sequencing. The probiotic potential such as acid and bile tolerance, digestive enzymes tolerance, cell surface hydrophobicity, cell autoaggregation, antibacterial activity against foodborne pathogens, blood haemolytic activity, and antibiotic susceptibility were determined. A total of 27 bacteria were isolated from bee bread and were identified as Lactobacillus spp., Fructobacillus fructosus, Leuconostoc mesenteroides, Enterococcus faecalis, and Bacillus spp. Most of the Bacillus spp. showed haemolytic abilities. L. musae SGMT17 and L. mindensis SGMT22 had similar antibacterial efficiency against tested pathogens in comparison to L. rhamnosus GG. All of the isolates showed high tolerance to $\mathrm{pH} 3,0.3 \%$ bile, pepsin, and pancreatin except for $\mathrm{F}$. fructosus strains. Lb. crustorum, Lb. mindensis and Lb. musae showed high hydrophobicity and autoaggregation ranging from 52.09 to $80.52 \%$ and 57.47 to $92.77 \%$, respectively, depending on the strain. All strains were susceptible to chloramphenicol, ampicillin and tetracycline, but varied for eight other clinically relevant antibiotics. From the result obtained, Lb. musae SGMT17 and Lb. crustorum SGMT20 showed the highest antibacterial activity and probiotic properties in the human in vitro digestive model. Future studies have to be conducted to assess other therapeutic value and safety of the selected bacteria in vivo.
\end{abstract}

Keyword: Probioticslactic; Acid bacteria; Bee bread; Bee pollen; Stingless bee; Anti bacterial activity; Heterotrigona itama 